\title{
Secondary Omental Torsion with Acute Appendicitis
}

\section{Pui WC*}

Department of Surgery, Kapit Hospital, Malaysia

*Corresponding author: Pui WC, Department of

Surgery, Kapit Hospital, J alan Mamora, 96800, Kapit, Sarawak, Malaysia

Received: November 24, 2017; Accepted: December 14, 2017; Published: December 20, 2017

\begin{abstract}
Omental torsion is a rare cause of acute abdomen with non-specific presentations mimicking other common pathologies. It is commonly found incidentally during surgery. This case presented as acute appendicitis and noted to have secondary omental torsion with simultaneous acute appendicitis intraoperatively. Open appendicectomy and partial omentectomy were performed. Ultrasonography and Computed Tomography can detect omental torsion preoperatively and is beneficial especially when patients have vague presentations. Hence, imaging can assist in planning of surgical treatment options.
\end{abstract}

Keywords: Omental torsion; Acute appendicitis; Acute abdomen; Appendicectomy

\section{Introduction}

Omental torsion was first described by Eitel in 1899 [1]. It is a rare cause of acute abdomen which mimics other commoner diagnoses and is commonly found intraoperatively. Omental torsion can be divided into primary, without any intra-abdominal pathology, or secondary torsion which happens alongside with other pre-existing conditions [2].

\section{Case Report}

This is a case of secondary omental torsion due to underlying acute appendicitis. A forty nine years old gentleman with no underlying illness presented with 3 days of right iliac fossa pain. He did not have anorexia, nausea or vomiting and was a febrile. Physical examination revealed tenderness over right iliac fossa at Mc Burney's point with rebound tenderness. His white blood cell was $12.4 \times 10^{9} / \mathrm{L}$, urinalysis was normal and KUB radiograph did not show any stones. Alvarado score was $6 / 10^{3}$. A diagnosis of acute appendicitis was made planned for open appendicectomy via Lanz's incision. Intra-operative finding was torsion of the right omentum (1080 degree) which was gangrenous. The distal omentum was adhered to the appendix which was inflamed. Patient underwent appendicectomy and partial omentectomy. He recovered well post-operatively and was discharged the next day. Histopathology examination confirmed the diagnosis of acute appendicitis (Figure 1).

\section{Discussion}

Omental torsion is a rare cause of acute abdomen which is difficult to diagnose. It usually occurs between the third to fifth decade and more commonly affects male compared to female with ratio of 2:1 [2]. Primary omental torsion is unipolar where the proximal omentum is fixed and the other parts remain free [2]. In contrast, secondary omental torsion has both fixed proximal and distal omentum hence termed bipolar [2]. The causes of secondary omental torsion are cysts, tumours, hernias, adhesions or intra-abdominal inflammations as in this case [2-4]. Omental torsions usually involved the distal right epiploic artery because the right side of the omentum is longer and thus more mobile and susceptible to torsion [5].
Clinical presentation of omental torsion is non-specific and depends on the causes and severity. The main symptom is sudden, localized right abdominal pain which intensify gradually [4]. Therefore, it is often misdiagnosed as appendicitis, cholecystitis, Meckel's diverticulitis, salphingitis or torsion ovarion cyst $[1,2,4,5]$. However, omental torsion is less associated with nausea, vomiting or anorexia as in our patient and the blood parameters are usually normal [4]. In late presentation, patients can have peritonitis and raised septic parameters due to omental gangrene $[2,4,5]$. That is why it is often found incidentally during surgery for acute abdomen.

Imaging is helpful in narrowing the differential diagnoses especially in equivocal cases. Ultrasonography may show a combination of hyperechoic, non-compressible mass with hypoechoic rim or a complex mass and intraperitoneal free fluid $[2,4,5]$. Computed Tomography (CT) scan will be more specific. Findings include a well circumscribed or oval fatty mass with heterogeneous attenuation, containing strands of twisted blood vessels whirling around a central rod, the "whirl sign" [2,4-6].

In this case, no imaging was done as the patient was treated as acute appendicitis. However, he did not have typical symptoms of acute appendicitis such as migratory right iliac fossa pain, anorexia, nausea or vomiting. Nevertheless, his tenderness was at Mc Burney's point and rebound tenderness was present. He also had leucocytosis with neutrophils shifted to the left. These totaled up to Alvarado score of six with possible diagnosis of acute appendicitis. Subsequently, we proceeded with surgery.

Surgical intervention with resection of non-viable omentum and correction of secondary pathology is the mainstay of treatment for omental torsion $[1,2,4-6]$. However, if diagnosis is confirmed with CT scan, there is a role for laparoscopic approach and even conservative management with antibiotics and analgesia, where the omentum will undergo regression ${ }^{6}$. Hence, imaging should be considered especially in patients who presented with right sided abdominal pain and vague symptoms or signs to make a clear diagnosis. We suggest that patients with right iliac fossa pain suspected of having acute appendicitis with Alvarado score below seven should have an imaging done to narrow down the diagnosis. If it showed omental torsion, then surgery can be 


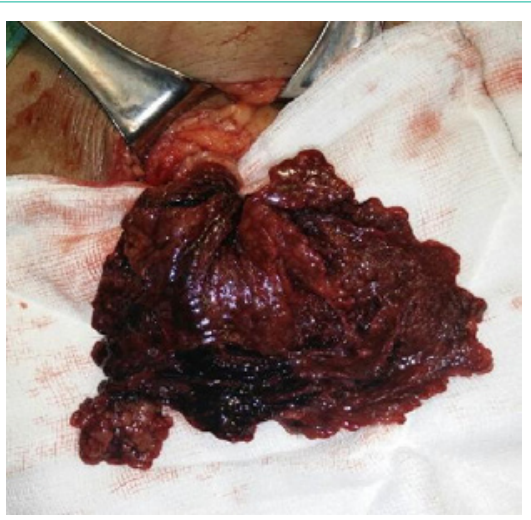

Figure 1: Right omentum twisted 1080 degree with gangrene. The distal portion adhering to the inflamed appendix had been separated and the omentum delivered out via the Lanz's incision.

planned properly such as type of open surgery incision, laparoscopic approach options and surgical instruments needed. Thus, imaging is certainly beneficial as surgeons can plan properly prior to surgery and patients can benefit from the advantages of laparoscopic approach or smaller open surgical incision which has less morbidity. When radiological modality is not readily available, diagnostic laparoscopy will naturally be beneficial in the diagnosis and resection of omental torsion in the same setting.

\section{Conclusion}

Being a rare entity of acute abdomen, omental torsion is difficult to diagnose as it mimics other common pathologies. Hence, the diagnosis of omental torsion should always be considered when treating acute abdomen especially with non specific right abdominal pain presentation. Further imaging such as abdominal CT scan or ultrasonography should be done in suspicious acute appendicitis with Alvarado score of below seven. Imaging can assist in treatment decision making and surgical planning preoperatively in omental torsion.

\section{References}

1. Eitel GG. Rare omental torsion. NY Med Rec. 1899; 55: 715-716.

2. Andreuccettti J, Ceribelli C, Manto O, Chiaretti M, Negro P, Tuscano D. Primary omental torsion (POT): a review of literature and case report. World Journal of Emergency Surgery. 2011; 6: 6.

3. Alvarado A. A practical score for the early diagnosis of acute appendicitis. Ann Emerg Med. 1986; 15: 557-564.

4. Tsironis A, Zikos N, Bali C, Pappas-Gogos G, Koulas S, Katsamakis N Primary torsion of the greater omentum: report of two cases and review of the literature. The Internet Journal of Surgery. 2008; 17: 239-246.

5. Joshi S, Cuthbert GA, Kerwat R. Omental torsion, a rare cause of acute abdomen. BMJ Case Rep. 2016.

6. Yu JS, Lee WS, Kim YH. Primary torsion of lesser omentum presented with acute abdomen and successfully managed with laparoscopic surgery. Chin Med J. 2016; 129: 1625-1626.
Austin J Surg - Volume 4 Issue 5 - 2017

ISSN : 2381-9030 | www.austinpublishing group.com

Pui. (C) All rights are reserved
Citation: Pui WC. Secondary Omental Torsion with Acute Appendicitis. Austin J Surg. 2017; 4(5): 1113. 